\title{
A Graph Theoretical Interpretation of Different Types of Energies of Elementary Particles, Atoms and Molecules
}

\author{
Jorge Galvez \\ Molecular Topology and Drug Design Research Unit, Department of Physical Chemistry, Faculty of Pharmacy, University of \\ Valencia, Burjasot (Valencia), Spain \\ Email: jorge.galvez@uv.es
}

How to cite this paper: Galvez, J. (2019) A Graph Theoretical Interpretation of Different Types of Energies of Elementary Particles, Atoms and Molecules. Open Journal of Physical Chemistry, 9, 33-50. https://doi.org/10.4236/ojpc.2019.92003

Received: January 17, 2019

Accepted: May 19, 2019

Published: May 22, 2019

Copyright () 2019 by author(s) and Scientific Research Publishing Inc. This work is licensed under the Creative Commons Attribution International License (CC BY 4.0).

http://creativecommons.org/licenses/by/4.0/

\section{(c) (i) Open Access}

\begin{abstract}
The present work illustrates a predictive method, based on graph theory, for different types of energy of subatomic particles, atoms and molecules, to be specific, the mass defect of the first thirteen elements of the periodic table, the rotational and vibrational energies of simple molecules (such as $\mathrm{H}_{2}^{+}, \mathrm{H}_{2}, \mathrm{FH}$ and $\mathrm{CO}$ ) as well as the electronic energy of both atoms and molecules (conjugated alkenes). It is shown that such a diverse group of energies can be expressed as a function of few simple graph-theoretical descriptors, resulting from assigning graphs to every wave function. Since these descriptors are closely related to the topology of the graph, it makes sense to wonder about the meaning of such relation between energy and topology and suggests points of view helping to formulate novel hypotheses about this relation.
\end{abstract}

\section{Keywords}

Graph Theory, Energy, Particles, Atoms, Molecules

\section{Introduction}

In an article published by the author a few years ago [1], it was proved that the masses of the 12 elementary particles of the standard model (fermions) and the vibrational energies of the hydrogen molecule can be accurately predicted using the same approach. Specifically, the formalism consisted of assigning graphs with increasing number of loops to the increasing energy levels, following the same pattern as the classical stationary waves.

Now, the aim is to extend this approach to other types of energies, like the mass defect of the less massive nuclides of the first 13 elements of the periodic 
table (between hydrogen and aluminium), the rotational and vibrational energies of diatomic molecules, such as $\mathrm{H}_{2}^{+}, \mathrm{H}_{2}, \mathrm{FH}$ and $\mathrm{CO}$, as well as the electronic energies of atoms and molecules (conjugated alkenes).

In all cases, the formalism proposed predicts accurately all these different energies, even improving the predictions made with the typical quantum approaches for such cases.

Altogether, these new results arise some interesting questions about the relation energy-topology.

\section{Assigning Graphs to Different Physical Systems. The Theoretical Framework}

In a previous paper, the author together with other colleagues proposed new ways to assign wave functions to graphs [2], which is exactly the reverse process proposed now.

The relation between wave functions and graphs is implicit in Heisenberg's matricial formulation of quantum mechanics [3], because for every matrix a graph of connections between its entries can be assigned. Moreover, Harary had studied the application of graphs to theoretical physics and chemistry [4]. More recently, the equivalence between wave functions and graphs has also been demonstrated [5].

It's not difficult to see that the transformation of wave functions into graphs has a double advantage: On the one hand, unlike most predictive models, it yields with pure mathematical and simple descriptors and not physical ones. On the other hand, the same model, namely the classical model of the stationary waves, can be used to predict very different types of energies (rotational, vibrational and electronic) at different levels (subatomic particles, atoms and molecules). Furthermore, it is well known that this classical pattern of interferences show the same shape as that of the quantum models, such as particle-in-a-box, rigid rotor, harmonic oscillator, free-electrons and Hückel. All are applicable to particles, atoms and molecules. Table 1 depicts, as an example, the equations of the wave functions and the associated energies for the particle in a box, the rigid rotor and the harmonic oscillator.

Although the equations complexity increases along with the model complexity

Table 1. Wave functions and energies for three systems: Particle in a box, rigid rotor and harmonic oscillator (Data from Levine, I. N. (1978a) Physical Chemistry. McGraw-Hill, New York, 550-554, 559-564).

\begin{tabular}{ccc}
\hline Model & Wave function & Energy \\
\hline $\begin{array}{c}\text { Particle in a } \\
\text { 1D-box }\end{array}$ & $\Psi(x)=\sqrt{2 / a} \sin \left(\frac{N \pi x}{a}\right)$ & $E=\frac{N^{2} h^{2}}{8 m a^{2}}, N=1,2,3, \cdots$ \\
$\begin{array}{c}\text { 1D-Rigid } \\
\text { Rotor }\end{array}$ & $\Psi(x)=(1 / 2 \pi)^{1 / 2} \mathrm{e}^{i N \phi}$ & $E=2 B(N+1), N=1,2,3$ \\
$\begin{array}{c}\text { Harmonic } \\
\text { oscillator }\end{array}$ & $\Psi(x)=\left(2^{v} v !\right)^{-1 / 2}(\alpha / \pi)^{1 / 4} \mathrm{e}^{-\alpha x^{2} / 2} H_{v}\left(\alpha^{1 / 2} x\right)$ & $E=h v_{e}(N+1 / 2), N=0,1,2,3, \cdots$ \\
\hline
\end{tabular}


(from the particle in a box to the harmonic oscillator), the topology of the wave functions, and hence of the graphs associated, remains unaltered.

This is the key basis for this work because one unique type of graphs accounts for any of the wave functions associated to every of the physical models mentioned above.

Hence, it were assigned graphs to each level starting with a simple graph without any loop (level $N=0$ ), and continuing by graphs containing $N$ loops, where $N$ is the number of the corresponding level. Table 2 shows the graph allocation for the 5 first levels of energy.

Once allocated the graphs, the formalism will be applied to the prediction of the following energies:

1) Mass defect. Applied onto the less massive nuclides of the first 13 elements of the periodic table (from hydrogen to aluminium).

2) Rotational energy. Rotational energies of the molecules of $\mathrm{H}_{2}, \mathrm{FH}$ and $\mathrm{CO}$.

3) Vibrational energy. Vibrational energies of the hydrogen molecule-ion, $\mathrm{H}_{2}^{+}, \mathrm{H}_{2}$ and $\mathrm{CO}$.

4) Electronic energy. The 1s-binding energy (ionization potential) of the first 13 elements of the periodic table (from hydrogen to aluminium). Additionally, it will be also predicted the HOMO-LUMO gap of conjugated alkenes.

In the predictions of atomic properties (mass defect and binding energy) it will be considered the atomic number as the topological level of each graph. Thus hydrogen would be $N=1$, helium $N=2$, and so forth.

\section{Material and Methods}

The topological indices were calculated using Dragon software [6] and the regression equations were obtained using BMDP software [7].

\section{Results and Discussion}

\subsection{Mass Defect}

Mass defect is defined as the mass loss produced when nuclei are formed from their constitutive particles (protons and neutrons) [8].

Table 2. Allocation of graphs to different energy levels.

\begin{tabular}{|c|c|c|}
\hline Graph & Graph's label & $\mathrm{N}$ \\
\hline & G1 & 0 \\
\hline & G2 & 1 \\
\hline & G3 & 2 \\
\hline & G4 & 3 \\
\hline & G5 & 4 \\
\hline
\end{tabular}


When correlating the mass defect for the most stable nuclides of the first thirteen elements of the periodic table, the best regression equation was:

$$
\begin{gathered}
M D(\mu \mathrm{u})=-10.47 \times S C B O+54 \\
N_{d}=13, R^{2}=0.9967, S E=4.79, F=3363
\end{gathered}
$$

The simple correlation with the mass number $(N n+Z)$ was also very good:

$$
\begin{gathered}
M D(\mu \mathrm{u})=-9.60 \times(N n+Z)+20.104 \\
N_{d}=13, R^{2}=0.9966, S E=4.86, F=3359
\end{gathered}
$$

where:

$M D=$ Mass defect (in $\mu \mathrm{u}$ ); $S C B O=$ Sum of count bond orders (overall number of edges in the graph); $N n=$ Number of neutrons; $N n+Z=$ Mass number; $N_{d}=$ Number of data; $R^{2}=$ Coefficient of determination; $S E=$ Standard error of estimate; $F=$ Fisher-Snedecor parameter.

Table 3 shows the values of the mass defects for the less massive nuclides of the first 13 elements of the periodic system. In annexed columns, the graph assigned to each nuclide, together with the values of atomic number $(Z)$, number of neutrons $(\mathrm{Nn})$ and mass defects observed (experimental) and calculated through Equations (1) and (2).

It is interesting to note that the regression with both, the atomic and the mass numbers, yielded worse fittings than SCBO $\left(R^{2}=0.9953,0.9966\right.$ and 0.9967 , respectively). Moreover, Equation (2) approaches the null value of hydrogen much better than Equation (3) (1.65 vs. 10.50), whereas the notable discrepancy for Be

Table 3. Values of Mass defects for the most stable nuclides of the thirteen first elements of the periodic table. The graph allocation and the values of $\mathrm{Z}, \mathrm{N}, \mathrm{SCBO}$ and the predicted values of MD are also included. Data from Moudgil, H.K. (2014). Textbook of Physical Chemistry, PHI Learning Pvt. Ltd, Delhi, 201.

\begin{tabular}{cccccccc}
\hline Element & $\boldsymbol{Z}$ & $\boldsymbol{N n}$ & $\boldsymbol{N n + Z}$ & $\mathrm{SCBO}$ & $\begin{array}{c}\text { Mass defect } \\
\text { (Experimental)* }\end{array}$ & $\begin{array}{c}\text { Cal. from } \\
\text { Equation (1) Equation (2) }\end{array}$ & $\begin{array}{c}\text { Cal. from } \\
\text { Equation }\end{array}$ \\
$\mathrm{H}$ & 1 & 0 & 1 & 5 & 0 & 1.6 & 10.5 \\
$\mathrm{He}$ & 2 & 1 & 3 & 6 & -8.3 & -8.8 & -8.7 \\
$\mathrm{Li}$ & 3 & 3 & 6 & 8 & -34.4 & -29.8 & -37.5 \\
$\mathrm{Be}$ & 4 & 3 & 7 & 10 & -40.4 & -50.7 & -47.1 \\
$\mathrm{~B}$ & 5 & 5 & 10 & 12 & -69.5 & -71.7 & -75.9 \\
$\mathrm{C}$ & 6 & 6 & 12 & 14 & -98.9 & -92.6 & -95.1 \\
$\mathrm{~N}$ & 7 & 7 & 14 & 16 & -112.4 & -113.5 & -114.4 \\
$\mathrm{O}$ & 8 & 8 & 16 & 18 & -137.0 & -134.5 & -133.6 \\
$\mathrm{~F}$ & 9 & 10 & 19 & 20 & -158.7 & -155.4 & -162.4 \\
$\mathrm{Ne}$ & 10 & 10 & 20 & 22 & -172.5 & -176.4 & -172.0 \\
$\mathrm{Na}$ & 11 & 12 & 23 & 24 & -200.3 & -197.3 & -200.8 \\
$\mathrm{Mg}$ & 12 & 12 & 24 & 26 & -212.8 & -218.3 & -210.4 \\
$\mathrm{Al}$ & 13 & 14 & 27 & 28 & -241.5 & -239.2 & -239.2 \\
\hline
\end{tabular}


can be explained by the exceptional fact that its more stable nuclide contains 5 and not 4 neutrons.

Equation (1) is particularly interesting as far as there are no theoretical equations for the mass defect, excepting a semi-empirical equation [9]. This semi-empirical equation contains, as the most representative term, the mass number, what agrees with the good correlation found from Equation (2).

\subsection{Rotacional Energy}

In this section the frequencies of transitions between rotational levels for three molecules, namely $\mathrm{H}_{2}, \mathrm{FH}$ and $\mathrm{CO}$, will be predicted.

The rotational frequencies for diatomic molecules are given by the quantum rigid and elastic rotors [10], according to the equations:

$$
\begin{aligned}
& v=2 B \times(N+1) \text { with } N=0,1,2,3,4, \cdots \quad \text { (Rigid rotor) } \\
& v=2 B \times(N+1)-4 D \times(N+1)^{3} \text { with } N=0,1,2,3,4, \cdots \quad \text { (Elastic rotor) }
\end{aligned}
$$

where $v=$ Rotational frequency. $B=$ Rotational Constant. $D=$ Centrifugal distortion constant.

The frequencies will be predicted for the spectral first lines of three different molecules: $\mathrm{H}_{2}, \mathrm{FH}$ and $\mathrm{CO}$ in their electronic and vibrational ground states.

Following are the results.

\subsubsection{Molecule of Hydrogen $\left(\mathrm{H}_{2}\right)$}

When correlating with $N$, it results:

$$
\begin{gathered}
v\left(\mathrm{~cm}^{-1}\right)=155.26 \times N+185.97 \\
N_{d}=7, R^{2}=0.9984, S E=14.83, F=3068
\end{gathered}
$$

where:

$v=$ Rotational frequency; $N=$ Topological-quantum level $(N=1,2,3, \cdots) ; N_{d}=$ Number of data; $R^{2}=$ Coefficient of determination; $S E=$ Standard error of estimate; $F=$ Fisher-Snedecor parameter.

The regression with PCR yielded:

$$
\begin{gathered}
v\left(\mathrm{~cm}^{-1}\right)=1264.26 \times P C R-1068.38 \\
N_{d}=7, R^{2}=0.9998, S E=5.16, F=25345
\end{gathered}
$$

where:

$v=$ Rotational frequency; $P C R=$ Ratio between graph's multiple path count and path count; $N_{d}=$ Number of data; $R^{2}=$ Coefficient of determination; $S E=$ Standard error of estimate; $F=$ Fisher-Snedecor parameter.

Following is Table 4, where is shown the comparison between the experimental and the predicted values, using Equations (3), (5), (6) and square $P C R$ (equation not shown).

From results in Table 4, it can be appreciated that Equations (3) and (5) (rigid rotor and $N$ ) predict better the lower values, whereas Equation (6) (PCR) predicts much better the values from $N=3$ on up. 
Table 4. Comparison between the experimental rotational frequencies of $\mathrm{H}_{2}$ and the calculated from Equations (4), (6), (7) and PCR squared. 'Data from: Edouard Hugo, Oskar Asvany, and Stephan Schlemmer. The journal of chemical physics, 130, 164302-1 to 17 (2009). doi: 10.1063/1.3089422.

\begin{tabular}{ccccc}
\hline $\begin{array}{c}\text { Experimental } \\
\text { rotational } \\
\text { frequencies }\left(\mathrm{cm}^{-1}\right)^{\mathbf{c}}\end{array}$ & $\begin{array}{c}\text { Calculated } \\
\text { from } \\
\text { Equation (5) }\end{array}$ & $\begin{array}{c}\text { Calculated from } \\
\text { Equation (6) }\end{array}$ & $\begin{array}{c}\text { Calculated from } \\
\text { Equation (3) } \\
\text { rigid rotor (2B = 170.4) }\end{array}$ & $\begin{array}{c}\text { Calculated } \\
\text { PCR }^{2}\end{array}$ \\
\hline 170.4 & 186.0 & 195.9 & 170.4 & 178.6 \\
339.3 & 341.2 & 328.6 & 340.8 & 327.4 \\
504.8 & 496.5 & 491.7 & 511.2 & 502.7 \\
665.4 & 651.8 & 654.8 & 681.6 & 669.7 \\
819.7 & 807.0 & 812.8 & 852.0 & 823.8 \\
965.7 & 962.3 & 965.8 & 1022.4 & 965.5 \\
1096.9 & 1117.5 & 1112.5 & 1192.8 & 1094.6 \\
\hline
\end{tabular}

\subsubsection{Molecule of Hydrogen Fluoride (HF)}

The corresponding equations for $N$ and $P C R$, were:

$$
\begin{gathered}
v\left(\mathrm{~cm}^{-1}\right)=38.75 \times N+48.65 \\
N_{d}=14, R^{2}=0.9994, S E=1.51, F=77125
\end{gathered}
$$

where:

$v=$ Rotational frequency; $N=$ Topological-quantum level $(N=1,2,3, \cdots) ; N_{d}=$ Number of data; $R^{2}=$ Coefficient of determination; $S E=$ Standard error of estimate; $F=$ Fisher-Snedecor parameter.

$$
\begin{gathered}
v\left(\mathrm{~cm}^{-1}\right)=348.85 \times P C R-310.58 \\
N_{d}=14, R^{2}=0.9989, S E=5.04, F=6931
\end{gathered}
$$

where:

$v=$ Rotational frequency; $P C R=$ Ratio between multiple path count and path count; $N_{d}=$ Number of data; $R^{2}=$ Coefficient of determination; $S E=$ Standard error of estimate; $F=$ Fisher-Snedecor parameter.

Table 5 presents the comparison between the experimental and predicted values. On the second column are the values of the differences between consecutive frequencies. From them, it's easy to see that the rigid rotor model is not followed because the differences become smaller and smaller.

\subsubsection{Molecule of Carbon Monoxide (CO)}

The corresponding equations were:

$$
\begin{gathered}
v\left(\mathrm{~cm}^{-1}\right)=7.61 \times N+11.45 \\
N_{d}=12, R^{2}=0.9999, S E=0.0009, F>100000
\end{gathered}
$$

where:

$v=$ Rotational frequency; $N=$ Topological-quantum level $(N=1,2,3, \cdots) ; N_{d}=$ Number of data; $R^{2}=$ Coefficient of determination; $S E=$ Standard error of 
Table 5. Experimental and calculated values of rotacional energy for the different levels of the molecule of HF. ${ }^{d}$ Data obtained from Mason, A.A.; Nielsen, A.H., Rotational spectrum of hydrogen fluoride: frequencies and linewidths, J. Opt. Soc. Am., 1967, 57, 1464.

\begin{tabular}{ccccc}
\hline $\begin{array}{c}\text { Experimental } \\
\text { frequency } \\
\left(\mathrm{cm}^{-1}\right)^{\mathrm{d}}\end{array}$ & $\Delta$ frequencies & $\begin{array}{c}\text { Calculated from } \\
\text { Equation }(3) \\
\left(\mathrm{B}=20.56 \mathrm{~cm}^{-1}\right)\end{array}$ & $\begin{array}{c}\text { Calculated from } \\
\text { Equation (7) }\end{array}$ & $\begin{array}{c}\text { Calculated from } \\
\text { Equation (8) }\end{array}$ \\
\hline 41.08 & 41.12 & 48.65 & 38.27 \\
82.19 & 41.11 & 82.24 & 87.40 & 74.90 \\
123.15 & 40.96 & 123.36 & 126.15 & 119.90 \\
164.01 & 40.85 & 164.48 & 164.90 & 164.90 \\
204.62 & 40.62 & 205.6 & 203.65 & 208.51 \\
244.93 & 40.31 & 246.72 & 242.40 & 250.72 \\
285.01 & 40.08 & 287.84 & 281.15 & 291.19 \\
324.65 & 39.64 & 328.96 & 319.90 & 330.26 \\
363.93 & 39.28 & 370.08 & 358.65 & 367.93 \\
402.82 & 38.89 & 411.2 & 397.40 & 404.21 \\
441.13 & 38.31 & 452.32 & 436.15 & 439.80 \\
478.94 & 37.81 & 493.44 & 474.90 & 474.33 \\
516.20 & 37.26 & 534.56 & 513.65 & 507.82 \\
\hline
\end{tabular}

estimate; $F=$ Fisher-Snedecor parameter.

$$
\begin{gathered}
v\left(\mathrm{~cm}^{-1}\right)=69.57 \times P C R-55.30 \\
N_{d}=12, R^{2}=0.9983, S E=1.21, F=4193
\end{gathered}
$$

where:

$v=$ Rotational frequency; $P C R=$ Ratio between multiple path count and path count; $N_{d}=$ Number of data; $R^{2}=$ Coefficient of determination; $S E=$ Standard error of estimate; $F=$ Fisher-Snedecor parameter.

Table 6 illustrates the comparison between the experimental and the calculated values.

In this case, the regression with $N$ is by far the best and practically coincides with that calculated using the rigid rotor equation.

Table 7 summarizes the results for all the three molecules.

Altogether it may be concluded that the excellent adjustment found for a single variable $(N)$, does not mean that experimental results are adjustable to the rigid rotor, since in that case, as deduced from Equation (3), the slope and intercept should take the same value. This is far to be true for $\mathrm{H}_{2}$ and HF, what is consistent with expressed in the literature [11]. On the contrary, CO fits pretty well the rigid rotor, what was to be expected considering that the centrifugal distortion must decrease as the molecular mass increases and hence that $\mathrm{CO}$ is less deformable than $\mathrm{FH}$ and $\mathrm{H}_{2}$.

The consequence is that the formalism proposed here, according to which the quantum level is equivalent to the graph's number of loops, remains consistent. 
Table 6. Experimental and calculated values of rotacional frequencies for the different levels of the molecule of CO. ${ }^{e}$ Data from: Harris, D.C. and M.D. Bertolucci, M.D. (1989). Symmetry and Spectroscopy. An Introduction to Vibrational and Electronic Spectroscopy. Dover Publications, 1989. ISBN 0-19-502001-4, 125.

\begin{tabular}{ccc}
\hline $\begin{array}{c}\text { Experimental } \\
\text { Frequencies }\left(\mathrm{cm}^{-1}\right)^{\mathbf{e}}\end{array}$ & $\begin{array}{c}\text { Calculated by } \\
\text { Equation }(9)\end{array}$ & $\begin{array}{c}\text { Calculated by } \\
\text { Equation (10) }\end{array}$ \\
\hline 11.43 & 11.45 & 10.67 \\
19.05 & 19.06 & 17.60 \\
26.67 & 26.67 & 26.11 \\
34.29 & 34.28 & 34.62 \\
41.91 & 41.89 & 42.86 \\
49.52 & 49.5 & 50.84 \\
57.13 & 57.11 & 58.50 \\
64.74 & 64.72 & 65.89 \\
72.34 & 72.33 & 73.01 \\
79.95 & 79.94 & 79.87 \\
87.55 & 87.55 & 86.60 \\
95.15 & 95.16 & 93.13 \\
\hline
\end{tabular}

Table 7. Summary of results for rotational frequencies.

\begin{tabular}{cccccc}
\hline \multicolumn{5}{c}{ SUMMARY of ROTATION } \\
\hline \multicolumn{7}{c}{$\mathrm{H}_{2}$} & \multicolumn{5}{c}{ HF } & CO & \\
\hline Model & $\mathbf{R}^{2}$ & Model & $\mathbf{R}^{2}$ & Model & $\mathbf{R}^{2}$ \\
\hline $\mathrm{N}$ & 0.9984 & $\mathrm{~N}$ & 0.9994 & $\mathrm{~N}$ & 0.9999 \\
$\mathrm{PCR}$ & 0.9980 & PCR & 0.9989 & PCR & 0.9983 \\
PCR2 & 0.9996 & PCR2 & 0.9998 & PCR2 & 0.9997 \\
Rigid rotor & 0.9964 & $\begin{array}{c}\text { Rigid rotor } \\
\left(2 \mathrm{~B}=41.12 \mathrm{~cm}^{-1}\right)\end{array}$ & 0.9988 & $\begin{array}{c}\text { Rigid rotor } \\
\left(2 \mathrm{~B}=11.43 \mathrm{~cm}^{-1}\right)\end{array}$ & 0.9999 \\
Elastic rotor & 0.9985 & Elastic rotor & 0.9998 & Elastic rotor & 1 \\
\hline
\end{tabular}

\subsection{Vibrational Energy}

The energies for the different vibrational levels of diatomic molecules are given by [12]:

$$
\begin{aligned}
& E_{v}=(N+1 / 2) h v_{e}, N=0,1,2,3, \cdots \text { Harmonic oscillator } \\
& E_{v}=(N+1 / 2) h v_{e}-(n+1 / 2)^{2} h_{e} v_{e} X_{e}, N=0,1,2,3, \cdots \text { nharmonic oscillator }
\end{aligned}
$$

where $v_{e}$ is the (classical) fundamental vibrational frequency, $X_{e}$ is the anharmonicity constant and $\mathrm{h}$ is the Planck constant.

Next are the results for the three molecules tested, namely $\mathrm{H}_{2}^{+}, \mathrm{H}_{2}$ and CO.

\subsubsection{Hydrogen Molecule-Ion $\left(\mathrm{H}_{2}^{+}\right)$}

The hydrogen molecule-ion is an ideal molecule to study since it is the most 
simple molecule possible and it has one single electron, so that the possible influence of interelectronic repulsion is overcome.

The correlation with $N$ is quite good as shown in Equation (14):

$$
\begin{aligned}
& v\left(\mathrm{~cm}^{-1}\right)=122.39 \times N+161.72 \\
& N_{d}=12, R^{2}=0.9972, S E=24.51, F=3566
\end{aligned}
$$

where:

$\nu=$ Vibrational frequency; $N=$ Topological-quantum level $(N=1,2,3, \cdots) ; N_{d}=$ Number of data; $R^{2}=$ Coefficient of determination; $S E=$ Standard error of estimate; $F=$ Fisher-Snedecor parameter.

Note that, as mentioned above, the first value for $N$ is here $N=1$ to take into account the existence of a residual vibratory energy.

Although there is a good correlation with $N$, a look at Figure 1 illustrates that the dependence is not linear but quadratic. In principle, this is consistent if there is a following of the model of anharmonic oscillator (Equation (14)). But see that in Equation (14) the intercept should be zero, what doesn't occur. Actually, the ratio between the ordinate and the slope $(23 / 149)$ is too large to be consistent with Equation (14). Of course, this difference could be explained considering other effects, such as the influence of rotational over vibrational energies, but, once more, this discrepancy provide value to the graph-theoretical formalism proposed by the author.

Anyway, the adjustment with $P C R$ is much better than the linear $N$ :

$$
\begin{gathered}
v\left(\mathrm{~cm}^{-1}\right)=1066.1 P C R-919.61 \\
N_{d}=12, R^{2}=0.9999, S E=5.25, F=77862
\end{gathered}
$$

where:

$v=$ Vibrational frequency; $P C R=$ Ratio between multiple path count and path count; $N_{d}=$ Number of data; $R^{2}=$ Coefficient of determination; $S E=$ Standard error of estimate; $F=$ Fisher-Snedecor parameter.

Table 8 illustrates the values of the vibrational frequencies of $\mathrm{H}_{2}^{+}$in its

Vibrational Frequency $\mathrm{H}_{2}+$ vs $\mathrm{N}$

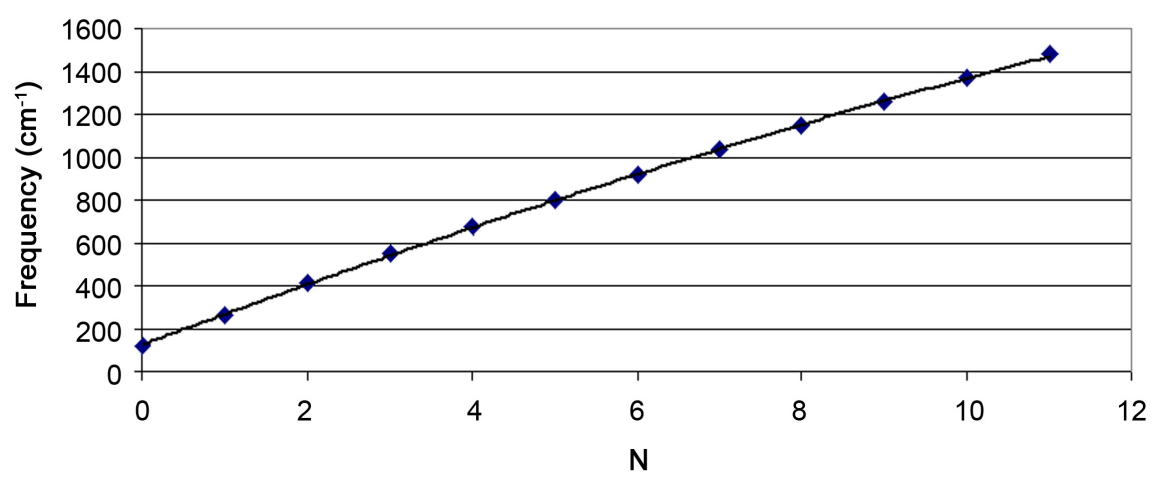

Figure 1. Vibrational frequency $\mathrm{H}_{2}+$ vs $\mathrm{N}$. 
electronic ground state for the first 11 levels of vibratory energy. The values calculated from Equations (14) and (15) are also included for comparison.

\subsubsection{Hydrogen Molecule $\left(\mathrm{H}_{2}\right)$}

In this case the aim is analysing the vibrational energy for the different levels $(N=0,1,2, \cdots)$.

The correlation with $N$ is quite good as seen in Equation (16):

$$
\begin{gathered}
v\left(\mathrm{~cm}^{-1}\right)=12416 \times N+18229 \\
N_{d}=12, R^{2}=0.9850, S E=16.62, F=665
\end{gathered}
$$

where:

$E_{V}=$ Vibrational energy; $N=$ Topological-quantum level $(N=1,2,3, \cdots) ; N_{d}=$ Number of data; $R^{2}=$ Coefficient of determination; $S E=$ Standard error of estimate; $F=$ Fisher-Snedecor parameter.

But the correlation with $P C R$ is much better:

$$
\begin{gathered}
v\left(\mathrm{~cm}^{-1}\right)=108125 \times P C R-91423 \\
N_{d}=12, R^{2}=0.9947, S E=9.83, F=1888
\end{gathered}
$$

where:

$v=$ Vibrational frequency; $P C R=$ Ratio between multiple path count and path count; $N_{d}=$ Number of data; $R^{2}=$ Coefficient of determination; $S E=$ Standard error of estimate; $F=$ Fisher-Snedecor parameter.

Similarly to $\mathrm{H}_{2}^{+}$, the dependence with $N$ is clearly quadratic, so that $R^{2}$ is

Table 8. Experimental vibrational frequencies for the molecule $\mathrm{H}_{2}^{+}$and comparison with the calculated from Equations (14) and (15). ${ }^{\mathrm{f}}$ Data obtained from Donald W. Rogers. Computational Chemistry Using the PC, 3rd Edition. ISBN: 978-0-471-42800-8, 302.

\begin{tabular}{ccc}
\hline $\begin{array}{c}\text { Experimental } \\
\text { frequencies for } \mathbf{H}_{2}^{+}\left(\mathrm{cm}^{-1}\right)^{\mathbf{f}}\end{array}$ & $\begin{array}{c}\text { Calculated from } \\
\text { Equation (14) }\end{array}$ & $\begin{array}{c}\text { Calculated from } \\
\text { Equation (15) }\end{array}$ \\
\hline 117 & 161 & 146 \\
265 & 288 & 258 \\
411 & 409 & 396 \\
548 & 530 & 533 \\
677 & 651 & 667 \\
800 & 772 & 796 \\
918 & 893 & 919 \\
1033 & 1014 & 1039 \\
1145 & 1135 & 1154 \\
1257 & 1256 & 1265 \\
1368 & 1377 & 1374 \\
1479 & 1498 & 1581 \\
\hline
\end{tabular}


virtually 1 what is consistent with the well known values for Xe. but for one single variable, $P C R$ is much better as can be seen comparing Equations (16) and (17).

Table 9 shows the experimental values of the vibration frequencies for the hydrogen molecule in its fundamental electronic state, together with the values calculated from Equations (16) and (17).

From these results, it is to note that $\mathrm{H}_{2}$, just like $\mathrm{H}_{2}^{+}$, does not fit well neither the harmonic oscillator nor the anharmonic oscillator. Therefore, the same arguments exposed for $\mathrm{H}_{2}^{+}$can be repeated for $\mathrm{H}_{2}$. Anyway, the dependence with $P C R$ is clearly linear and better than the ones of conventional models if equations having the same number of variables are compared. It is also to be noted that other topological indices (such as ESpm02r) led to better results but PCR was left for comparative purposes.

\subsubsection{Molecule of Carbon Monoxide (CO)}

The correlation with $N$ is quite good as illustrated in Equation (18):

$$
\begin{gathered}
v\left(\mathrm{~cm}^{-1}\right)=2040.57 \times N+1238.2 \\
N_{d}=10, R^{2}=0.9998, S E=105.77, F=30709
\end{gathered}
$$

where:

$v=$ Vibrational frequency; $N=$ Topological-quantum level $(N=1,2,3, \cdots) ; N_{d}=$ Number of data; $R^{2}=$ Coefficient of determination; $S E=$ Standard error of estimate; $F=$ Fisher-Snedecor parameter.

The equation for PCR IS:

$$
\begin{gathered}
v\left(\mathrm{~cm}^{-1}\right)=17217 \times P C R-16016 \\
N_{d}=10, R^{2}=0.9995, S E=141.40, F=17179
\end{gathered}
$$

Table 9. Experimental Vibrational frequencies for the molecule $\mathrm{H}_{2}$ and comparison of the calculated through Equations (16) and (17). ${ }^{\mathrm{g}}$ Data taken from J.M. Hernando. Problemas de Quimica Fisica. Graficas A. Martin S.A. ISBN: 84-400-6995-2, 179.

\begin{tabular}{ccc}
\hline $\begin{array}{c}\text { Vibrational energy observed } \\
\left(\mathrm{cm}^{-1}\right)^{\mathbf{g}}\end{array}$ & $\begin{array}{c}\text { Calc. from } \\
\text { Equation (16) }\end{array}$ & $\begin{array}{c}\text { Calc. from } \\
\text { Equation (17) }\end{array}$ \\
\hline 4547 & 9150 & 8352 \\
13,265 & 15,358 & 14,029 \\
21,481 & 21,566 & 21,003 \\
29,195 & 27,774 & 27,977 \\
36,407 & 33,982 & 34,735 \\
43,117 & 40,190 & 41,276 \\
49,331 & 46,398 & 47,548 \\
55,037 & 52,606 & 53,603 \\
60,237 & 58,814 & 59,442 \\
64,931 & 65,022 & 65,064 \\
69,137 & 71,230 & 70,579 \\
72,836 & 77,438 & 75,931 \\
\hline
\end{tabular}


where:

$v=$ Vibrational frequency; $P C R=$ Ratio between multiple path count and path count; $N_{d}=$ Number of data; $R^{2}=$ Coefficient of determination; $S E=$ Standard error of estimate; $F=$ Fisher-Snedecor parameter.

In this case the fitting to the harmonic oscillator continues to be poor, although the adjustment with the anharmonic (in this case matches with that of $N^{2}$ ) is practically perfect.

Table 10 shows the comparison between the experimental values and those calculated from Equations (20) and (21).

From these overall results of vibrational frequencies, it can be concluded that the graph-theoretical model $(P C R)$ is better for small molecules, such as $\mathrm{H}_{2}^{+}$ and $\mathrm{H}_{2}$ whereas the conventional approaches are better for larger molecules, such as CO.

That conclusion is evident just watching Table 11, where it is summarized all the results.

Table 11 summarizes the results for the three molecules.

Table 10. Experimental vibrational frequencies for the molecule of $\mathrm{CO}$ and comparison of the calculated from Equations (18) and (19). ' Data obtained from Bernhard Schrader, Ed. (1995). Infrared and Raman Spectroscopy: Methods and Applications. DOI: $10.1002 / 9783527615438,520-525$.

\begin{tabular}{ccc}
\hline $\begin{array}{c}\text { Vibrational energies CO } \\
\left(\mathrm{cm}^{-1}\right)^{\mathbf{h}}\end{array}$ & $\begin{array}{c}\text { Frequation Calc. from } \\
\text { Equation (18) }\end{array}$ & $\begin{array}{c}\text { Frequation Calc. From } \\
\text { Equation (19) }\end{array}$ \\
\hline 1082 & 1238 & 1201 \\
3227 & 3279 & 3009 \\
5345 & 5319 & 5230 \\
7438 & 7360 & 7451 \\
9505 & 9400 & 9603 \\
11,545 & 11,441 & 11,686 \\
13,560 & 13,482 & 13,683 \\
15,548 & 15,522 & 15,612 \\
17,511 & 17,563 & 17,471 \\
19,447 & 19,603 & 19,262 \\
\hline
\end{tabular}

Table 11. Summary of results for the vibrational frequencie.

\begin{tabular}{cccccc}
\hline \multicolumn{5}{c}{ SUMMARY VIBRATIONAL ENERGIES } \\
\hline & $\mathbf{H}_{2}^{+}$ & \multicolumn{5}{c}{$\mathrm{H}_{2}$} & \multicolumn{2}{c}{ CO } \\
\hline Variable & $\mathbf{R}^{2}$ & Variable & $\mathbf{R}^{2}$ & Variable & $\mathbf{R}^{2}$ \\
\hline $\mathrm{N}$ & 0.9972 & $\mathrm{~N}$ & 0.9850 & $\mathrm{~N}$ & 0.9997 \\
$\mathrm{PCR}$ & 0.9993 & $\mathrm{PCR}$ & 0.9928 & $\mathrm{PCR}$ & 0.9995 \\
$\mathrm{PCR}^{2}$ & 0.9994 & $\mathrm{PCR}^{2}$ & 0.9998 & $\mathrm{PCR}^{2}$ & 0.9996 \\
$\begin{array}{c}\text { Harmonic } \\
\text { oscillator }\end{array}$ & 0.9832 & $\begin{array}{c}\text { Harmonic } \\
\text { oscillator }\end{array}$ & 0.9655 & $\begin{array}{c}\text { Harmonic } \\
\text { oscillator }\end{array}$ & 0.9994 \\
$\mathrm{~N}^{2}$ & 0.9983 & $\mathrm{~N}^{2}$ & 1,0000 & $\mathrm{~N}^{2}$ and Anharmonic \\
oscillator & 1,0000 \\
\hline
\end{tabular}




\subsection{Electronic Energy}

In this section, different sorts of electronic energies for atoms and molecules will be analysed.

\subsubsection{Electronic Binding Energy of Atoms}

Electronic binding energy in atoms also usually referred to as ionization potential [13] is defined as the energy necessary to remove the electron from the atom. In this case we'll correlate the binding energies for the 1s-electrons of the thirteen first elements of the periodic system.

The regresión equation with quadratic $N$ was almost perfect:

$$
\begin{gathered}
E B E-1 s(\mathrm{eV})=10.60 \times N^{2}-20.43 \times N+23.79 \\
N_{d}=12, R^{2}=0.9999, S E=4.91, F=43500
\end{gathered}
$$

where:

$E B E=$ Electronic binding energy (eV) of the first atomic level (1S); $N=$ Topological-quantum level $(N=1,2,3, \cdots) ; N_{d}=$ Number of data; $R^{2}=$ Coefficient of determination; $S E=$ Standard error of estimate; $F=$ Fisher-Snedecor parameter.

Table 12 shows the values of EBEs for the 13 first elements of the periodic table, along with the values calculated from Equation (20).

Given the nature of the correlated property, it is clear that $N$ might be identified as $Z$ (atomic number), and since the ionization potential for electrons of a given electronic level, $n$, is [14].

$$
E=-\frac{Z^{2} e^{2}}{2 a_{0} n^{2}}
$$

Table 12. Experimental and calculated (Equation (22)) values of EBE-1s for the first 13 elements of periodic table. ${ }^{\mathrm{i}}$ Data from: $\underline{\text { http://xdb.lbl.gov/Section1/Table_1-1.pdf. }}$

\begin{tabular}{ccc}
\hline Atom & EBE-1S (eV) (observed) & Calculated from Equation $(20)$ \\
\hline $\mathrm{H}$ & 13.6 & 14.0 \\
$\mathrm{He}$ & 24.6 & 25.3 \\
$\mathrm{Li}$ & 54.7 & 57.9 \\
$\mathrm{Be}$ & 111.5 & 111.7 \\
$\mathrm{~B}$ & 188.0 & 186.6 \\
$\mathrm{C}$ & 284.2 & 282.7 \\
$\mathrm{~N}$ & 409.9 & 400.1 \\
$\mathrm{O}$ & 543.1 & 538.6 \\
$\mathrm{~F}$ & 696.7 & 698.3 \\
$\mathrm{Ne}$ & 870.2 & 879.2 \\
$\mathrm{Na}$ & 1070.8 & 1081.4 \\
$\mathrm{Mg}$ & 1303.0 & 1304.7 \\
$\mathrm{Al}$ & 1559.6 & 1549.2 \\
\hline
\end{tabular}


where $n=$ Atomic level. As in this case $n=1$ (1S) for all the levels, the binding energy depends on $Z^{2}$. Hence a good correlation with $Z^{2}$ should be obtained, something that actually happens $\left(R^{2}=0.9986\right)$.

However, although with $Z^{2}$ there is an acceptable correlation $\left(R^{2}=0.9986\right)$ the dependence is not proportional, as it should be according to Equation (21). This allows us to do the assignment of $N$ as the topological level and interpret Equation (20) as a virial-like development in powers of $N$ (see Figure 2).

\subsubsection{Electronic Energies in Molecules: Homo-Lumo Gap (HLG) of Conjugated Alkenes}

In this paragraph the resonance energies of conjugated alkenes will be predicted. Two different formalisms are followed: In the first the type G graphs (Table 3) will be used to characterize the energy levels, while in the second, the conventional graph-molecular presentation (graphs type $\mathrm{M}$, where $\mathrm{M}=$ molecular). In the $M$ representation, each molecule is represented by a graph containing the same number of alternated loops as alternated double bonds have the molecule.

For instance, the graph allocated to ethylene is $(\mathrm{M} 1):=$, and for butadiene (M2): $»$, etc.

1) Formalism using graphs type G:

The best correlation was for:

$$
\begin{gathered}
H L G(\mathrm{eV})=8.03 \times \text { Vindex }-1.29 \\
N_{d}=9, R^{2}=0.9979, S E=0.074, F=3392
\end{gathered}
$$

where:

$H L G=$ HOMO-LUMO gap $(\mathrm{eV}) ;$ Vindex $=$ Balaban Vindex; $N_{d}=$ Number of data; $R^{2}=$ Coefficient of determination; $S E=$ Standard error of estimate; $F=$ Fisher-Snedecor parameter.

The Balaban V index [15] is a typical information index which takes into account the bond multiplicity.

\section{Electron Binding Energy vs N+1}

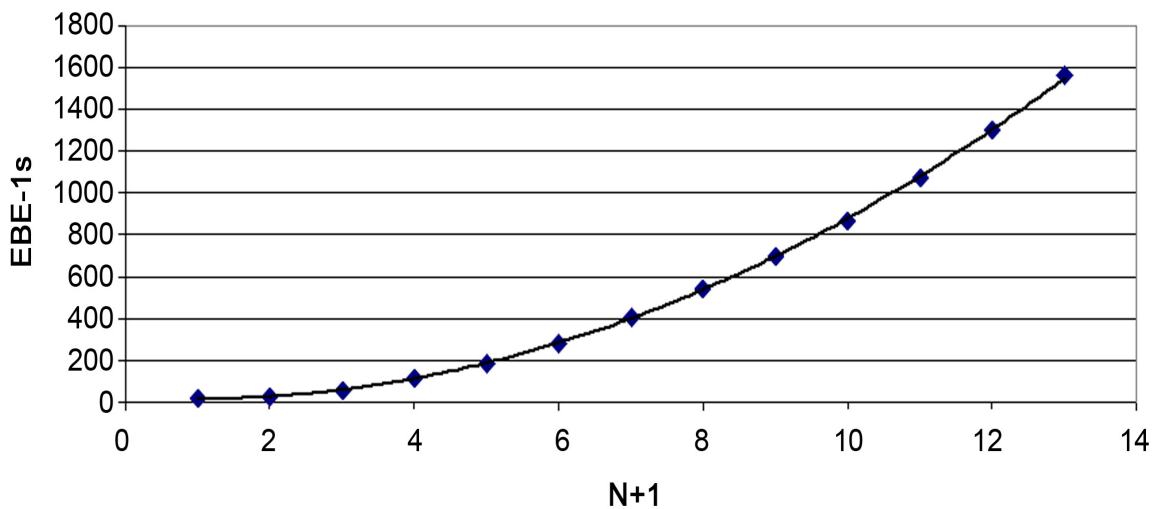

Figure 2. Election binding energy vs. $\mathrm{N}+1$. 
Table 13. Comparison of the experimental values of HLG with the obtained from Equation (24) 'Data from: Advanced Lab Course: UV-Vis Absorption Spectroscopy. Humboldt-Universität zu Berlin. Mathematisch-Naturwissenschaftliche Fakultät. Institut für Physik.Physik von Makromolekülen UV-VIS absorption characterization of (macro) molecular solutions (see web page).

\begin{tabular}{|c|c|c|c|}
\hline Alkene & $\begin{array}{c}\text { Graph } \\
\text { allocated }\end{array}$ & $\begin{array}{c}\text { HLG }(\mathrm{eV}) \\
\text { (Experimental) }\end{array}$ & $\begin{array}{c}\text { HLG (calculated } \\
\text { from Equation (22)) }\end{array}$ \\
\hline ethylene & $\mathrm{G} 2$ & 7.52 & 7.44 \\
\hline 1,3-butadiene & G3 & 5.72 & 5.73 \\
\hline 1,3,5-hexatriene & G4 & 4.63 & 4.77 \\
\hline 1,3,5,7-octatetraene & G5 & 4.08 & 4.15 \\
\hline 1,3,5,7,9-decacapentaene & G6 & 3.72 & 3.72 \\
\hline 1,3,5,7,9,11-dodecahexaene & G7 & 3.41 & 3.39 \\
\hline $1,3,5,7,9,11,13$-tetradecaneeptene & G8 & 3.18 & 3.15 \\
\hline $1,3,5,7,9,11,13,15$-esadecaneoctaene & G9 & 3.03 & 2.95 \\
\hline $1,3,5,7,9,11,13,15,17$-octadecanenonaene & G10 & 2.78 & 2.78 \\
\hline
\end{tabular}

Table 13 shows the comparison between the observed and the calculated (Equation (22)) Values of HLG.

Although not shown here, it is important to note that this model (Equation (22)) improved both the free-electron model and the Hückel model [16] $\left(R^{2}=\right.$ 0.9958 and $\mathrm{R}^{2}=0.9959$, respectively).

2) Formalism using graphs type M:

The best equation was:

$$
\begin{gathered}
H L G(\mathrm{eV})=-1.73 \times \operatorname{Espm} 02 x+10.23 \\
N_{d}=9, R^{2}=0.9985, S E=0.063, F=4759
\end{gathered}
$$

where:

$H L G=$ HOMO-LUMO gap (eV);ESpm02x = Spectral moment $\# 2$ of the edge adjacency matrix weighted by edge degrees; $N_{d}=$ Number of data; $R^{2}=$ Coefficient of determination; $S E=$ Standard error of estimate; $F=$ Fisher-Snedecor parameter.

Table 14 shows the experimental and the calculated values for HLG.

Noteworthy from the outcomes that the graph theoretical approach proposed by the author, either using graphs type $\mathrm{G}$ or type $\mathrm{M}$, improves the predictions of HLG done using conventional quantum methods, particularly the free-electrons and Hückel.

Table 15 illustrates, as a summary, the assignment of graphs and the values of the molecular graph-theoretical descriptors included in the equations of this work. The aim is ease the reader the reproduction of the results outlined throughout the work. 
Table 14. Observed and calculated values for HLG of the set of conjugated alkenes.

\begin{tabular}{cccc}
\hline Alkene & $\begin{array}{c}\text { Graph } \\
\text { allocated }\end{array}$ & HLG (eV) & $\begin{array}{c}\text { HLG calculated } \\
\text { form Equation (23) }\end{array}$ \\
\hline Ethylene & M1 & 7.52 & 7.44 \\
1,3-butadiene & M2 & 5.72 & 5.65 \\
$1,3,5$-hexatriene & M3 & 4.63 & 4.79 \\
$1,3,5,7$-octatetraene & M4 & 4.08 & 4.22 \\
$1,3,5,7,9$-decacapentaene & M5 & 3.72 & 3.79 \\
$1,3,5,7,9,11$-dodecahexaene & M6 & 3.41 & 3.44 \\
$1,3,5,7,9,11,13$-tetradecaneeptene & M7 & 3.18 & 3.15 \\
$1,3,5,7,9,11,13,15$-esadecaneoctaene & M8 & 3.03 & 2.91 \\
$1,3,5,7,9,11,13,15,17$-octadecanenonaene & M9 & 2.78 & 2.69 \\
\hline
\end{tabular}

Table 15. Values of the most significant topological indices in the prediction of the different energies studied in this work.

\begin{tabular}{cccccccc}
\hline $\begin{array}{c}\text { Graph } \\
\text { allocated } \\
\text { (Type G) }\end{array}$ & $\begin{array}{c}\text { N (number } \\
\text { of nodes) }\end{array}$ & SCBO & PCR & Vindex & ESpm08x & $\begin{array}{c}\text { Graph } \\
\text { allocated } \\
\text { (Type M) }\end{array}$ & $\begin{array}{c}\text { ESpm02x (for } \\
\text { M-graphs) }\end{array}$ \\
\hline G1 & 0 & 5 & 1 & 1.088 & 10.171 & M1 & 1.610 \\
G2 & 1 & 6 & 1.105 & 1.088 & 11.094 & M2 & 2.640 \\
G3 & 2 & 8 & 1.234 & 0.875 & 11.186 & M3 & 3.130 \\
G4 & 3 & 10 & 1.363 & 0.755 & 11.342 & M4 & 3.470 \\
G5 & 4 & 12 & 1.488 & 0.678 & 11.484 & M5 & 3.710 \\
G6 & 5 & 14 & 1.609 & 0.624 & 11.608 & M6 & 3.910 \\
G7 & 6 & 16 & 1.725 & 0.584 & 11.719 & M7 & 4.080 \\
G8 & 7 & 18 & 1.837 & 0.553 & 11.818 & M8 & 4.220 \\
G9 & 8 & 20 & 1.945 & 0.528 & 11.909 & M9 & 4.340 \\
G10 & 9 & 22 & 2.049 & 0.507 & 11.992 & M10 & 4.480 \\
G11 & 10 & 24 & 2.151 & 0.49 & 12.069 & M11 & 4.607 \\
G12 & 11 & 26 & 2.25 & 0.475 & 12.14 & M12 & 4.724 \\
G13 & 12 & 28 & 2.346 & 0.462 & 12.206 & M13 & 4.832 \\
G14 & 13 & 30 & 2.440 & 0.45 & 12.269 & M14 & 4.936 \\
\hline
\end{tabular}

\section{Conclusions}

The assignment of graphs to the energy levels of different systems (from elementary particles up to molecules) allows an excellent prediction of parameters such as masses of elementary particles, mass defects of stable nuclei, electronic energies of atoms and rotational, vibrational and electronic energies of molecules.

In particular, the wave functions associated with quantum-mechanical models, like particle in a box, rigid rotor or harmonic oscillator, are assimilated to simple graphs whose topology (number of nodes) coincides with that of the standing waves, as for instance those that appear on the strings of musical in- 
struments. This assimilation is justified by the Heisenberg's matricial formulation, by virtue of which every quantum object (for example the wave functions) can be associated with a matrix and this, in turn, with a graph.

For almost all cases, the formalism proposed improves the predictions of the conventional quantum-mechanical models (particle in a box, rigid rotor, harmonic oscillator, model of free electrons and Hückel), through the use of simple topological indices.

Moreover, in those cases where a very good correlation is found simply thanks to the number of nodes in the graph $(\mathrm{N})$, it is demonstrated that the solution is not trivial, since it does not coincide with what expected from the aforementioned conventional quantum models.

Since such a diverse type of energy can be expressed as a function of simple topological indices which, given their mathematical nature, are not dependent on energy, formalisms such as the one proposed here, may open suggestive pathways of discussion about the relations between energy and topology.

\section{Acknowledgements}

Authors acknowledge the MINECO (Spanish Ministry of Economy, Industry and Competitivity) Project: "Desarrollo de nuevas herramientas para el control de oidios" (AGL2016-76216-C2-2-R).

\section{Conflicts of Interest}

The author declares no conflicts of interest regarding the publication of this paper.

\section{References}

[1] Galvez, J. (2012) A Graph-Theoretical Approach to Calculating Vibrational Energies of Atomic and Subatomic Systems. Open Journal of Physical Chemistry, 2, 204-211. https://doi.org/10.4236/ojpc.2012.24028

[2] Galvez, J., Garcia-Domenech, R. and de Julian-Ortiz, J.V. (2006) Assigning Wave Functions to Graphs: A Way to Introduce Novel Topological Indices. Communications in Mathematical and in Computer Chemistry, 56, 509-518.

[3] Heisenberg, W. (1925) Quantum-Theoretical Re-Interpretation of Kinematic and Mechanical Relations. Zeitschrift fur Physik, 33, 879-893. https://doi.org/10.1007/BF01328377

[4] Harary, F. (1967) Graph Theory and Theoretical Physics. Academic Press, New York.

[5] Filk, T. (2006) Relational Interpretation of the Wave Function and a Possible Way around Bell's Theorem. International Journal of Theoretical Physics, 45, 1166-1180. https://doi.org/10.1007/s10773-006-9125-0

[6] Talete Srl Milano (2006) Dragon. http://www.talete.mi.it

[7] Dixon, W.J., Brown, M.B., Engelman, L. and Jennrich, R.I. (1990) BMDP. 7M Package. University of California Press, San Francisco.

[8] Choppin, G., Rydberg, J. and Lidjenzin, J.O. (1995) Radiochemistry and Nuclear Chemistry. 2nd Edition, Academic Press, Oxford, 45-46. 
[9] Von Weizsäcker, C.F. (1935) Zur Theorie der Kernmassen. Zeitschrift für Physik, 96, 431. https://doi.org/10.1007/BF01337700

[10] Vemulapalli, K.G. (1993) Physical Chemistry. Prentice Hall, Upper Saddle River, 402.

[11] Jennings, D.A., Evenson, K.M., Zink, L.R., Demuynck, C., Destombes, J.L., Lemoine, B. and Johns, J.W.C. (1987) High Resolution Spectroscopy of HF from 40 to $1100 \mathrm{~cm}^{-1}$ : Highly Accurate Rotational Constants. Journal of Molecular Spectroscopy, 122, 477-480. https://doi.org/10.1016/0022-2852(87)90021-X

[12] Moudgil, H.K. (2014) Textbook of Physical Chemistry. PHI Learning Pvt. Ltd., Delhi, 201.

[13] Gross, J. (2011) Mass Spectrometry. 2nd Edition, Springer-Verlag, Berlin-Heidelberg, 25. https://doi.org/10.1007/978-3-642-10711-5

[14] Anchordoqui, L.A. (2015) Symmetries and the Fundamental Forces of Nature II. 9. http://lehman.edu/faculty/anchordoqui/300-QM.pdf

[15] Balaban, A.T. and Balaban, T.S. (1991) New Vertex Invariants and Topological Indices of Chemical Graphs Based on Information on Distances. Journal of Mathematical Chemistry, 8, 383-397. https://doi.org/10.1007/BF01166951

[16] Levine, I.N. (1978) Physical Chemistry. McGraw-Hill, New York, 632-634. 